\title{
Correction: DLPFC transcriptome defines two molecular subtypes of schizophrenia
}

Elijah F. W. Bowen', Jack L. Burgess ${ }^{1}$, Richard Granger ${ }^{1}$, Joel E. Kleinman² and C. Harker Rhodes ${ }^{1}$

\section{Correction to: Translational Psychiatry \\ https://doi.org/10.1038/s41398-019-0472-z \\ Article published online 9 May 2019}

The original Article was missing an important note at the end of the "Implications of increased statistical power and druggable targets" heading. This has been added, and Joel Kleinman's affiliation information has also been amended.

Published online: 12 June 2019 